\title{
THE EFFECT OF TRIETHYLTIN ON MITOCHONDRIAL SWELLING*
}

\author{
K. E. Moore $†$ and T. M. Brody \\ Department of Pharmacology, University of Michigan Medical School, Ann Arbor, \\ Michigan
}

(Received 22 July 1960)

\begin{abstract}
Triethyltin induces swelling of isolated rat liver mitochondria suspended in sucrose or $\mathrm{KCl}$ media. This effect is not seen with brain mitochondria. Triethyltin swelling is only minimally reduced by EDTA or amytal but is prevented by 2:4-dinitrophenol, cyanide or salicylate. The addition of adenosine tri- or diphosphate causes reversal of the swelling; this effect is potentiated by $\mathbf{M n}^{2}$ but not by $\mathrm{Mg}^{2-1}$. The reversal of thyroxine-induced swelling by ATP is blocked by triethyltin, perhaps via an inhibition of the hydrolysis of adenosine triphosphate. The relationship between in vitro effects of triethyltin on mitochondria and the toxic actions of the drug in the whole animal are discussed.
\end{abstract}

IN THF past few years many investigators have studied the phenomenon of mitochondrial swelling. ${ }^{1-8}$ Much of the interest has centered around the role of phosphate bond energy and oxidative phosphorylation in the maintenance of mitochondrial integrity. Substrates, cofactors, ions and more recently agents which uncouple oxidative phosphorylation have been examined for their effects upon mitochondrial swelling. Generally, there has been no correlation between uncoupling action and mitochondrial permeability; some uncoupling agents enhance while others protect against mitochondrial swelling. ${ }^{2,3}$

The present work will demonstrate the effects of an organo-tin compound on mitochondrial permeability. This compound, triethyltin (TET) is an extremely toxic agent, ${ }^{9,10}$ a potent uncoupler of oxidative phosphorylation ${ }^{11}$ and an inhibitor of mitochondrial adenosine triphosphatases (ATPases). ${ }^{12}$ These studics were initiated with the view that such experiments might yield information pertinent to the elucidation of the phenomenon of mitochondrial swelling and further shed some light upon the mechanism of toxicity of the organo-tin compounds.

\section{METHODS}

Mitochondria were isolated from the livers of unstarved Holtzman female rats weighing $200-250 \mathrm{~g}$ by the method of Hogeboom et al.$^{13}$ Brain mitochondria were prepared as described by Brody and Bain. ${ }^{14}$ The homogenization fluid was $0.25 \mathrm{M}$ sucrose in those experiments in which swelling was determined in $\mathrm{KCl}$-tris (hydroxymethyl) aminomethane (Tris) medium. When swelling was measured in sucrose-Tris medium, $0.3 \mathrm{M}$ sucrose and $0.02 \mathrm{M}$ Tris buffer $\mathrm{pH} 7.4$ was used for the preparation and resuspension of the mitochondria. The mitochondria were prepared so that 1.0 $\mathrm{ml}$ of the final suspension contained the mitochondria from $0.5 \mathrm{~g}$ of liver. This stock suspension was kept at $0-2{ }^{\circ} \mathrm{C}$ and used within $2-3 \mathrm{hr}$ after preparation. Swelling experi-

* Preliminary studies were reported in Fed. Proc. 19, 139 (1960).

$\uparrow$ Present address: Department of Pharmacology, Dartmouth Medical School, Hanover, N.H. 
ments were performed in KCl-Tris $(0.125 \mathrm{M} \mathrm{KCl}-0.02 \mathrm{M}$ Tris, $\mathrm{pH} \mathrm{7.4)}$ or in the sucrose-Tris medium described above. Other additions are designated in the individual figures. The total volume in each cuvette was $3.0 \mathrm{ml}$ and additions of the mitochondrial stock solution $(0.08-0.1 \mathrm{ml})$ were made prior to mixing and reading. With this mitochondrial suspension an initial optical density reading of from 0.500 to 0.600 was obtained at $520 \mathrm{~m} \mu$ in the Beckman model DU spectrophotometer. After the initial reading the optical density values were recorded at 5 or $10 \mathrm{~min}$ intervals for $30 \mathrm{~min}$. Incubation was at room temperature $\left(22-24^{\circ} \mathrm{C}\right)$. Additions to the cuvettes during the experiments were made in small volumes to minimize optical density changes.

\section{RESULTS}

\section{Effect of the suspending medium on TET-induced mitochondrial swelling}

Figs. 1 and 2 illustrate the ability of TET to induce swelling of isolated liver mitochondria in both KCl-Tris and sucrose-Tris buffer. Mitochondrial swelling, as evidenced by a decrease in the optical density values of a mitochondrial suspension, could

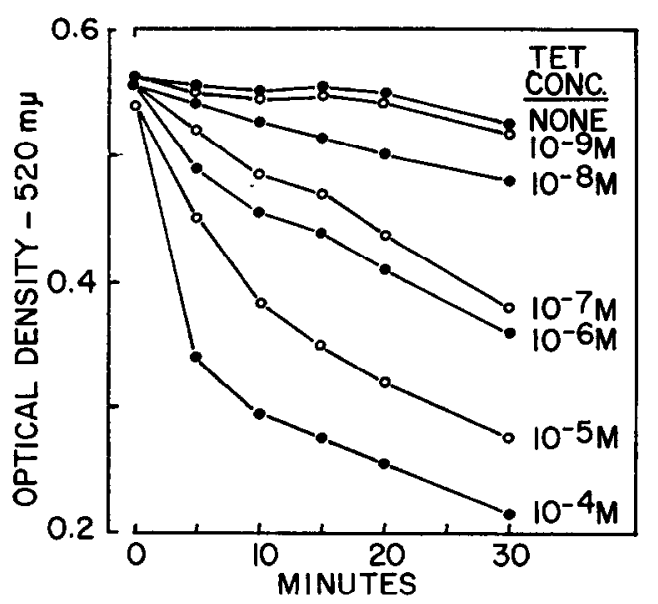

FIg. 1. Effect of TET on mitochondria suspended in $0.125 \mathrm{M} \mathrm{KCl}$ and $0.02 \mathrm{M}$ Tris buffer, $\mathrm{pH} 7 \cdot 4$.

always be obtained with concentrations of TET as low as $10^{-7} \mathrm{M}$ in $\mathrm{KCl}-\mathrm{Tris}$ media and $10^{-6} \mathrm{M}$ in sucrose-Tris. As the concentration of TET was increased, the effects upon swelling became more pronounced.

The osmolarity of the sucrose solution influenced the induction of swelling by TET. A sucrose concentration of $0.6 \mathrm{M}$ reduced both the spontaneous and TET-induced swelling; but even in this medium some swelling was observed when TET was present (Fig. 3). In a solution of $0 \cdot 15 \mathrm{M}$ sucrose, the spontaneous swelling became very pronounced so that the effects of TET appeared to be less prominent.

Since the mitochondria appeared to be approximately ten times more sensitive to the swelling effect of TET in $\mathrm{KCl}$-Tris buffer, most experiments were performed in this medium, although qualitatively similar results could be obtained when the experiments were repcatcd in sucrose-Tris buffer. All ATP-reversal studies were carried out in the $\mathrm{KCl}$-Tris medium.

The optimum $\mathrm{pH}$ value for swelling with TET was between 7.5 and 8.0 , which is approximately the same as that observed for spontaneous or thyroxine-induced swelling, but higher than that seen with reduced glutathione. ${ }^{15}$ 


\section{Ethylenediaminetetra-acetic acid (EDTA) on TET swelling}

Since it has been observed that many divalent heavy metal ions can cause mitochondrial swelling ${ }^{3,8}$ it was deemed advisable to determine whether TET-induced swelling could be the result of impurities of inorganic tin rather than an effect of the organic compound per se. As illustrated in Fig. 4 the addition of EDTA (final concentration, $10^{-4} \mathrm{M}$ ) at the beginning of the experiment or after $10 \mathrm{~min}$ only slightly reduced both TET-induced swelling and spontaneous swelling of mitochondria. Higher concentrations of EDTA $\left(10^{-3}\right.$ to $\left.10^{-2} \mathrm{M}\right)$ failed to block the TET-induced swelling to any significant extent.

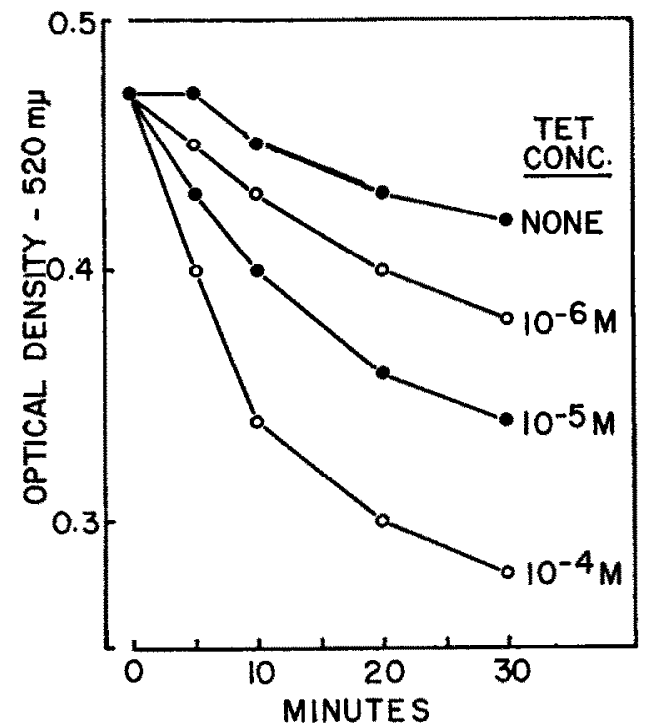

Fig. 2. Effect of TETlon mitochondria suspended in $0.3 \mathrm{M}$ sucrose and $0.02 \mathrm{M}$ Tris buffer, $\mathrm{pH} 7.4$.

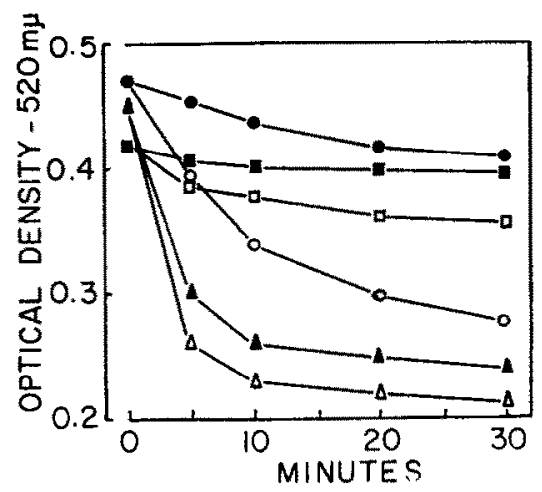

FIG. 3. Effect of sucrose concentration on spontaneous and TET-induced mitochondrial swelling. control; and $\bigcirc-10^{-4} \mathrm{M}$ TET; in $0.3 \mathrm{M}$ sucrose. $\Delta$-control; and $\triangle-10^{-4} \mathrm{M} \mathrm{TET}$; in $0.15 \mathrm{M}$ sucrose. $\square-c o n t r o l$; and $\square-10^{-4} \mathrm{M}$ TET; in $0.6 \mathrm{M}$ sucrose.

\section{Blockade of adenosine triphosphate $(A T P)$ reversal of thyroxine swelling}

Lehninger and co-workers have shown that thyroxine is capable of inducing a pronounced mitochondrial swelling in a $\mathrm{KCl}$-Tris medium which can be reversed by the addition of ATP. ${ }^{15,}{ }^{16}$ It was also reported that such uncoupling agents as azide, 
$p$-hydroxymercuribenzoate (formerly known as p-chloromercuribenzoate (PCMB)) and gramicidin inhibit the ATP reversal of swelling induced by thyroxine. As shown in Fig. 5 TET is also capable of blocking this reversal. This effect by TET was always prominent at a concentration of $10^{-6} \mathrm{M}$ TET but only slight inhibition was evident at TET concentrations as low as $10^{-7} \mathrm{M}$. It was also observed that when TET was added during the process of thyroxine-induced swelling, there was only a slight additional swelling above that usually seen with thyroxine alone. Thus it would appear that the blockade of ATP reversal was a true inhibition and not merely a reflection of a greater swelling produced by the TET which might have masked the ATP reversal.

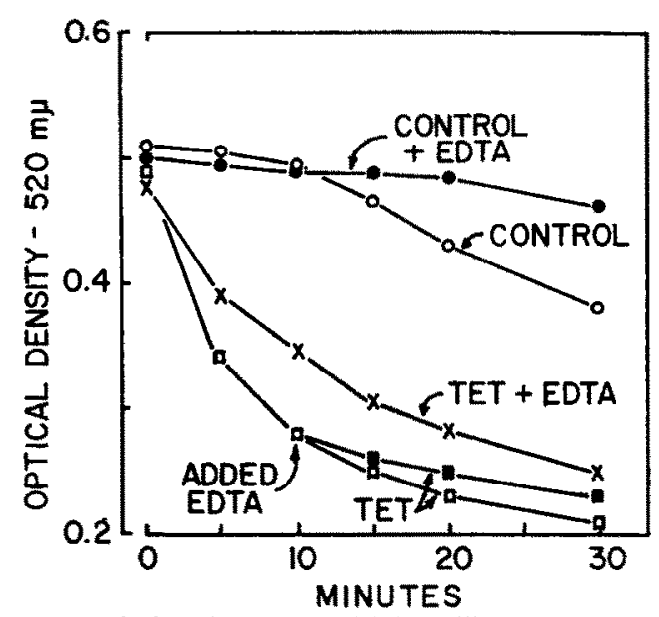

FIG. 4. Effect of EDTA on TET-induced mitochondrial swelling. The test system contained $0 \cdot 125 \mathrm{M}$ $\mathrm{KCl}$ and $0.02 \mathrm{M}$ Tris buffer, $\mathrm{pH} 7.4$ and $10^{-4} \mathrm{M}$ EDTA and $10^{-4} \mathrm{M}$ TET added as indicated.

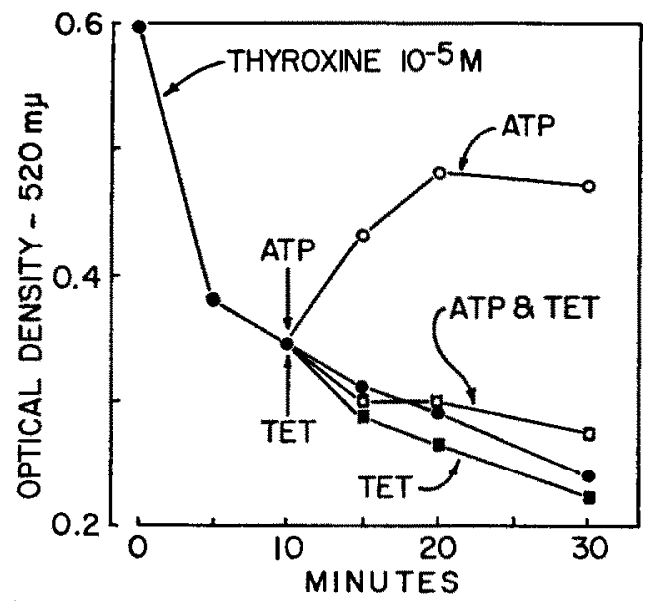

Frg. 5. Effect of TET on ATP reversal of thyroxine-induced swelling. The test system contained 0.125 $\mathrm{M} \mathrm{KCl}$ and $0.02 \mathrm{M}$ Tris buffer, pH 7.4; $1 \times 10^{-5} \mathrm{M}$ thyroxine and $3 \times 10^{-3} \mathrm{M}$ ATP and $1 \times 10^{-6}$ $M$ TET (final concentrations) added at $10 \mathrm{~min}$ as indicated by arrows.

4. Reversal of TET swelling by $A T P$ and $A D P$

As demonstrated in Fig. 6 ATP has the ability to reverse mitochondrial swelling produced by low concentrations of TET $\left(10^{-7} \mathrm{M}\right)$. The addition of ATP to mitochondrial suspensions containing higher TET concentrations $\left(10^{-6}-10^{-4} \mathrm{M}\right)$ was not 
effective in causing reversal. In Fig. 7 it may be observed that adenosine diphosphate (ADP) was less effective than ATP. The addition of adenosine monophosphate (AMP) could not reverse the TET-induced mitochondrial swelling. These data may be contrasted with the actions of the nucleotides on thyroxine-induced mitochondrial swelling, where ATP appears to be specific in its ability to reverse swelling. In our experiments the onset was immediate upon ATP addition, while the reversal seen with ADP was often slower in developing.

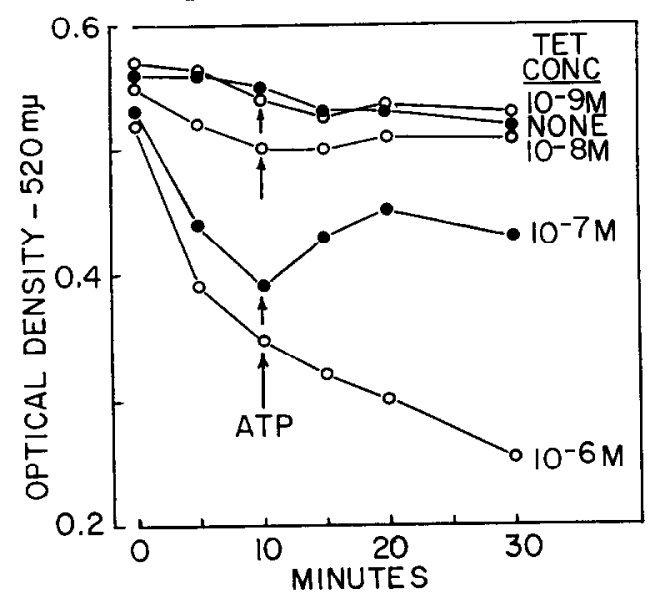

Fig. 6. Effect of ATP in reversing TET-induced swelling. The test system contained $0.125 \mathrm{M} \mathrm{KCl}$ and $0.02 \mathrm{M}$ Tris buffer, $\mathrm{pH} 7 \cdot 4$. ATP $\left(3 \times 10^{-3} \mathrm{M}\right.$, final concentration) was added as indicated by arrows.

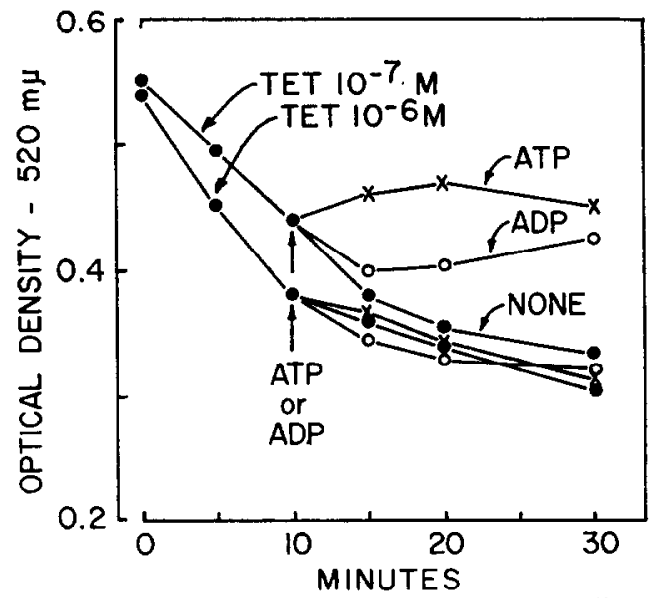

FIG. 7. Reversal of TET-induced swelling by ADP and ATP. Test medium contained 0.125 M KCl and $0.02 \mathrm{M}$ Tris buffer, $\mathrm{pH} 7.4$ with concentrations of TET as shown. ATP and ADP (final concentrations, $3 \times 10^{-3} \mathrm{M}$ ) added as indicated by arrows. TET concentrations are final.

\section{Effect of manganese on TET-induced swelling}

It has been reported that mitochondrial swelling caused by many agents may be reversed by ATP if $\mathrm{Mg}^{2}$ । or $\mathrm{Mn}^{2+}$ are present in the medium. The reversal of thyroxine swelling by ATP requires neither $\mathrm{Mg}^{2+}$ nor $\mathrm{Mn}^{2+}$ ions. ${ }^{17}$ If $\mathrm{Mn}^{2+}$ is added to the medium, ATP is now able to reverse the TET-induced swelling when the organo-tin compound is present in concentrations as high as $10^{-4} \mathrm{M}$ (Fig. 8). Thus, the addition of $\mathrm{Mn}^{2+}$ markedly potentiates the reversing action of ATP. A similar potentation was 
also observed with the addition of $\mathrm{Mn}^{2+}$ ions in studies with ADP. At a concentration of $3 \times 10^{-3} \mathrm{M}, \mathrm{MgCl}_{2}$ had no such effect on the reversal of TET swelling even though this ion has been reported to aid in reversing the swelling induced by other agents ${ }^{17}$.

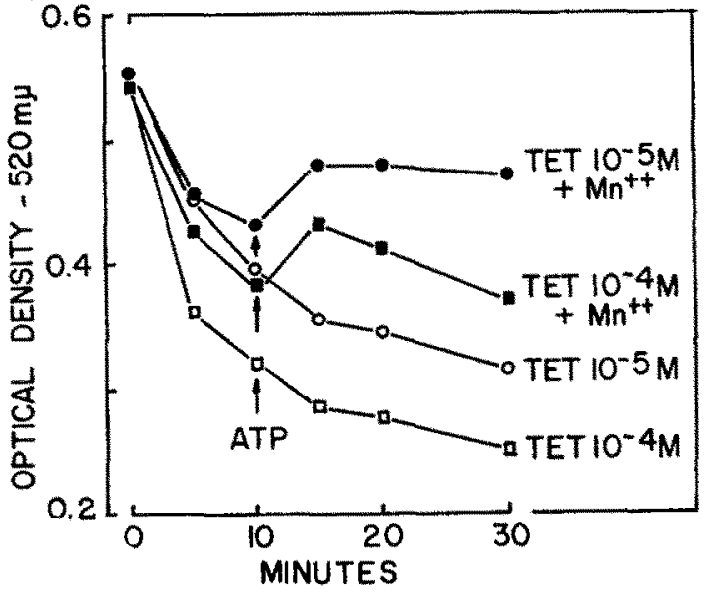

Fig. 8. Effect of manganese on TET-induced swelling and its reversal. The test medium was $0.125 \mathrm{M}$

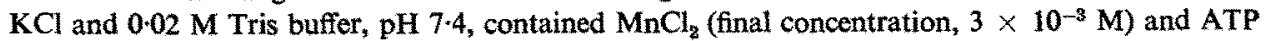
added at $10 \mathrm{~min}$ (final concentration $3 \times 10^{-3} \mathrm{M}$ ). All TET concentrations are final.

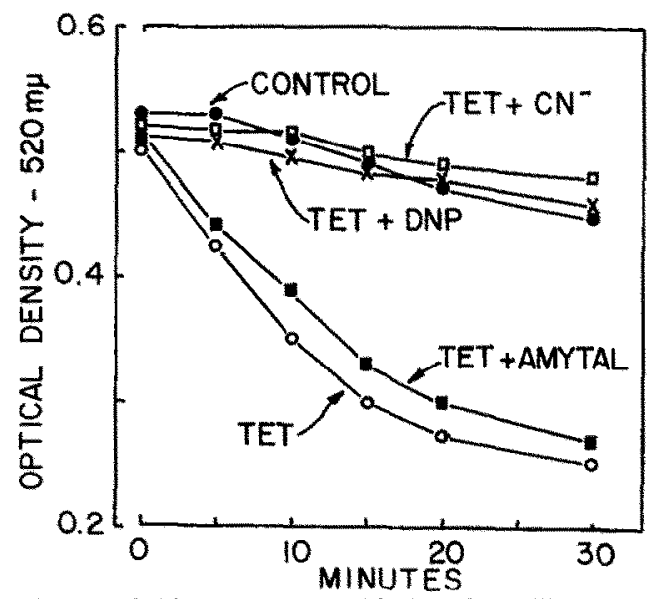

FIG. 9. The effect of respiratory inhibitors on TET-induced swelling. The test medium contained $0.125 \mathrm{M} \mathrm{KCl}$ and $0.02 \mathrm{M}$ Tris buffer, pH 7.4 with $2 \times 10^{-3} \mathrm{M} \mathrm{NaCN}, 2 \times 10^{-3} \mathrm{M}$ amytal and 3.3

$\times 10^{-5} \mathrm{M}$ 2:4-dinitrophenol; $1 \times 10^{-4} \mathrm{M}$ TET added as indicated. All concentrations are final.

\section{Effect of electron transport chain blockade}

In an attempt to determine the role played by the various constituents of the transport chain several studies concerned with the effects of electron transport chain inhibitors on mitochondrial swelling have been reported..$^{18-20}$ As seen in Fig. 9, blockade of the electron transport chain by $\mathrm{NaCN}$ resulted in a protective effect upon TET swelling whereas no protection was observed with sodium amytal. The addition of 2:4-dinitrophenol (DNP) effectively prevented swelling induced by $10^{-4} \mathrm{M}$ TET in sucrose-Tris and $\mathrm{KCl}$-Tris media. Although not shown in the figure, $3 \times 10^{-3} \mathrm{M}$ sodium salicylate also prevented TET-induced swelling. Agents capable of blocking electron flow through the hydrogen transport system have been reported to block 
mitochondrial swelling produced by such substrates as succinate, glutamate and $\beta$-hydroxybutyrate. TET does not block swelling induced by glutamate but rather enhances the swelling response, so that the effects of substrate and TET on swelling appear to be additive (Fig. 10). Similar effects were observed when swelling was induced by succinate.

\section{Studies on brain mitochondria}

The marked effect of TET on the nervous system and its ability to produce edema in this tissue ${ }^{21,22}$ prompted us to study the effects of TET on isolated brain mitochondria. It has been difficult to induce swelling in vitro by any means in brain mitochondria, ${ }^{23}$ although a recent report has indicated that under special conditions slight swelling can apparently be produced in brain mitochondria. ${ }^{24}$ We have been unable to observe any swelling in isolated rat brain mitochondria when TET was added in vitro.

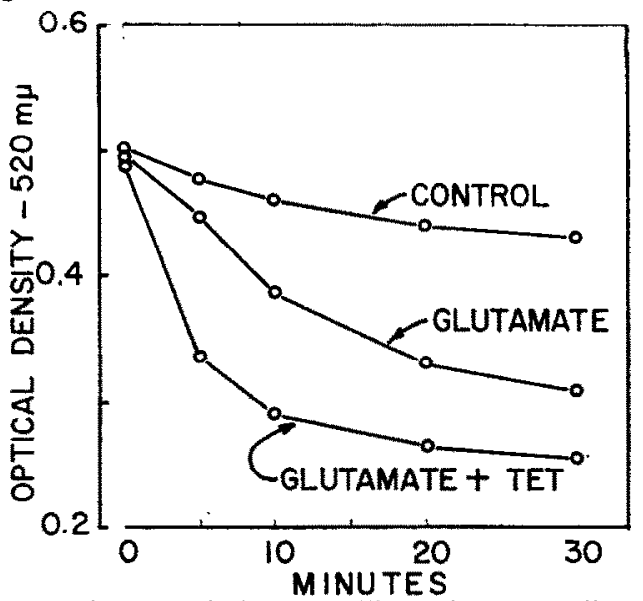

FIG. 10. The effect of TET on glutamate-induced swelling. The test medium contained $0 \cdot 125 \mathrm{M} \mathrm{KCl}$ and $0.02 \mathrm{M}$ Tris buffer, $\mathrm{pH} 7.4$ and TET $\left(1 \times 10^{-4} \mathrm{M}\right)$ and glutamate $\left(3 \times 10^{-3} \mathrm{M}\right)$ as indicated. All concentrations are final.

DISCUSSION

TET, at low concentrations, induces swelling of isolated rat liver mitochondria suspended in $\mathrm{KCl}$-Tris or sucrose-Tris buffers. Sucrose has been reported to be an unfavorable medium for studying mitochondrial swelling since sucrose solutions of varying osmolarity modify this phenomenon. ${ }^{15}$ In the present study, however, qualitatively similar effects were observed in both $\mathrm{KCl}$ and sucrose media; the mainr difference was that the TET induced swelling was more pronounced and could be reversed by ATP in the KCl-Tris buffer.

Mitochondrial swelling has been produced by a number of metal ions such as $\mathrm{Hg}^{2+}, \mathrm{Ag}^{+}$and $\mathrm{Zn}^{2+},{ }^{3,17}$ The addition of EDTA reduces the swelling produced by these metals and by many other agents indicating that some other property of EDTA in addition to its chelating ability is responsible for this protection. ${ }^{17}$ The mitochondrial swelling induced by TET, unlike the swelling produced by many other agents, ${ }^{3}$ is not blocked by concentrations of EDTA as high as $10^{-2} \mathrm{M}$. It would appear therefore that the mitochondrial swelling observed following the addition of TET is the result of the action of the organo-tin compound per se and not to any impurity of inorganic tin ions since EDTA would be expected to protect the mitochondria from sủch metallic impurities. 
There has been a great deal of work concerned with the effects of thyroxine on mitochondrial swelling. Dickens and Salmony ${ }^{4}$ and more recently Lehninger et al. ${ }^{15}$ have shown that thyroxine-induced swelling can be reversed by the addition of ATP to the medium. Water which enters the mitochondria during swelling is apparently extruded following the addition of ATP. To explain this phenomenon Lehninger ${ }^{16}$ postulated an active contractile mechanism in the mitochondrial membrane that is associated with the hydrolysis of ATP. Since ATP reversal of swelling is blocked by azide, arsenate and PCMB he suggested that the contraction could be related in some way with the mechanism of oxidative phosphorylation. However, since ATP reversal of thyroxine swelling is not inbibited by cyanide, antimycin A nor DNP, neither respiration nor coupled phosphorylation is apparently required for the contractile mechanism. It was demonstrated that $10^{-6} \mathrm{M}$ TET blocks the ATP reversal of thyroxine swelling. Since TET, like azide and PCMB is capable of inhibiting both the 2:4-dinitrophenol and the magnesium-activated mitochondrial ATPases ${ }^{12,25}$ it is possible that blockade of the ATP enzymic hydrolysis rather than the inhibition of some reaction occurring during oxidative phosphorylation may account for the ability of TET to block the ATP reversal of thyroxine induced swelling. It is interesting to note that TET inhibits ATPase activity at the same concentration $\left(10^{-6} \mathrm{M}\right.$ ) which blocks ATP reversal of thyroxine swelling; at this concentration TET has only minimal effects on oxidative phosphorylation.

ATP, and to a lesser extent ADP, reverse mitochondrial swelling induced by low concentrations of TET $\left(10^{-2} \mathrm{M}\right)$. At higher concentrations of TET these nucleotides cannot reverse the resultant swelling unless manganese ions are present. Both ATP and ADP, the latter probably by conversion to ATP via adenylate kinase, may activate some contractile process in the mitochondrial membrane. How the manganese ions markedly potentiate the actions of ATP is not presently known although this ion has been reported to have remarkable stabilizing actions on mitochondria. ${ }^{3}, 18$

The role of the electron transport chain on mitochondrial swelling has been studied by several investigators. ${ }^{18-20}$ Hunter $^{18}$ proposed that substances that promote electron transport should promote swelling while substances inhibiting the transport of electrons along the chain should block swelling. Exceptions to this hypothesis are those agents which have a direct effect on the mitochondrial membrane. The present study does not conflict with this hypothesis since TET, which has no effect on electron transfer ${ }^{26}$ is incapable of blocking glutamate or succinate-induced swelling. Furthermore, blockade of the electron transport chain by cyanide prevents TET-induced swelling.

It is difficult to assess the significance of TET-induced mitochondrial swelling in relation to the actions of this compound on other mitochondrial functions. Tapley ${ }^{3}$ concluded that since thyroxine caused mitochondrial swelling at a concentration lower than that required to uncouple oxidative phosphorylation, the ability of thyroxine to alter mitochondrial membrane integrity was the primary action of the hormone on the mitochondrion. This might also be stated for the actions of TET since swelling is produced at a concentration of TET which is lower than that which effectively uncouples phosphorylation. However, since in the oxidative phosphorylation studics other factors (sucrose, ATP, ADP, $\mathrm{Mg}^{2+}$, phosphate) are present which have pronounced actions on mitochondrial stability it is difficult to compare the relative effectiveness of TET on the two systems. The ability of TET to cause swelling probably explains the mitochondrial requirement for diphosphopyridine nucleotide which was observed during the oxidative phosphorylation studies. ${ }^{12}$ 
Although TET caused swelling of liver mitochondria in vitro, no swelling was observed in liver mitochondria from TET-treated rats. It should be emphasized that the medium in which swelling is measured in vitro is much different from that of the intact cell. Intracellular swelling is undoubtedly controlled by a dynamic balance of substrates, cofactors and ions so that marked mitochondrial swelling within the cell is probably produced only when an extreme imbalance of these factors occurs. ${ }^{27}$ Presumably such an imbalance does occur in the brain during the course of TET toxicity since a recent study utilizing the electron microscope has shown that the glial cells and mitochondria in the brains of TET treated mice are swollen. ${ }^{22}$ In the light of this finding it is paradoxical that no swelling is observed when brain mitochondria are treated in vitro with TET.

Although mitochondrial swelling has some properties in common with other membrane transport phenomena ${ }^{15}$ it is difficult to assess the importance of this effect in relation to the actions of TET upon the whole animal. Some effects of TET that might be pertinent to the present study are the observations that this compound produces cerebral swelling ${ }^{92}, 22$ and inhibits water and glucose transport in the rat intestine. ${ }^{28}$

Acknowledgements-The technical assistance of Mr. John Fuscaldo in these studies is gratefully acknowledged. Triethyltin acetate was obtained from Mr. E. L. Weinberg, Metal and Thermit Company, Rahway, N.J. Rats used in this study were obtained from the Upjohn Company, Kalamazoo, Michigan through the courtesy of Dr. Burton Baker. These studies were aided by a contract between the office of Naval Research, Department of the Navy and the University of Michigan, NR 303-439.

\section{REFERENCES}

1. J. RaAflaur, Helv. Physiol. Acta 11, 142 (1953).

2. A. L. LeHningre, Enzymes: Units of Biological Structure and Function (Edited by O. H. Gaebler) p. 217. Academic Press, New York (1956).

3. D. F. TAPLEY, J. Biol. Chem. 222, 325 (1956).

4. F. DiCKeNS and D. SALMONY, Biochem. J. 64, 645 (1956).

5. J. B. Chappell and G. D. Greville, Nature, Lond. 182, 813 (1958).

6. C. A. Price, A. Fonnesu and R. E. Davis, Biochem. J. 64, 754 (1956).

7. H. TedeschI and D. L. HLarRIS, Biochim. Biophys. Acta 28, 392 (1958).

8. F. E. Hunter, Jr., and L. Ford, J. Biol. Chem. 216, 347 (1955),

9. T. Alayouanine, L. Derobert and S. Thieffry, Rev. Neurol. 98, 85 (1958).

10. H. B. Stoner, J. M. Barnes and J. I. DUF, Brit, J. Pharmacol. 10, 16 (1955).

11. W. N. ALDRIDGE, Biochem. J. 69, 367 (1958).

12. K. E. Moore and T. M. Brody, Biochem. Pharmacol. 6, 13 (1961).

13. G. H. Hogeboom, W. C. Schneider and G. E. Pallade, J. Biol. Chem. 172, 619 (1948).

14. T. M. Brody and J. A. BAIN, J. Biol. Chem. 195, 685 (1952).

15. A. L. Lehninger, B. L. RAY and M. SChnerder, J. Biophys. Biochem. Cytol. 5, 97 (1959).

16. A. L. Lehninger, J. Biol. Chem. 234, 2187 (1959).

17. A. L. Lehninger, J. Biol. Chem. 234, 2465 (1959).

18. F. E. Hunter, JR., J. F. LeVY, J. Fink, B. SChUTZ, F, GuerRA and A. Hurwitz, J, Biol. Chem. 234, $2176(1959)$.

19. L. M. Corwin and M. N. LipsetT, J. Biol. Chem. 234, 2453 (1959),

20. J. B. ChapPel. and G. D. Greville, Biochim. Biophys. Acta 38, 483 (1960).

21. P. N. MAGeE, H. B. Stoner and J. M. BARnes, J. Path. Bact. 73, 107 (1957).

22. R. M. TORACK, R. D. Terry and H. M. Zimmerman, Amer. J. Path. 36, 273 (1960).

23. D. F. TAPLEY and C. COOPER, Nature, Lond. 178, 1119 (1956).

24. D. R. DAHL, R. J. JACoBs and F. E. SAMson, JR., Amer. J. Physiol. 198, 467 (1960).

25. P. Stekevirz, H. Löw, L. Ernster and O. Lindererg, Biochim. Biophys. Acta 29, 378 (1958).

26. W. N. Aldridge, and J. E. CRLMer, Biochem. J. 61, 406 (1955),

27. K. E. MOORE and T. M. Brody, Amer. J. Physiol. 198, 676 (1960).

28. B. J. PARsons, J. Physiol. 148, 117 (1959), 